\title{
UNIQUE ERGODICITY FOR CERTAIN RANDOM TRANSLATIONS
}

\author{
BENTON JAMISON AND ROBERT SINE
}

\begin{abstract}
Spatially dependent convex combinations of a pair of irrationally related translations on $\mathbf{R}$ are shown to admit at most one invariant probability. The only condition on the coefficient functions is measurability and essential positivity.
\end{abstract}

A particle moves on $(-\infty, \infty)$ at times $t=0,1, \ldots$ If it is at $x$ at time $t=n$, it moves to $x+a$ with probability $p(x)$ and to $x-b$ with probability $q(x)=1-p(x)$. We assume that both $a$ and $b$ are positive, so that the particle moves back and forth. We want to avoid the case where the possible positions of the particle are contained in a translate of a discrete subgroup of $(-\infty, \infty)$, so we assume that $a / b$ is irrational. We also assume, of course, that the function $p$ is Borel measurable on $(-\infty, \infty)$ into $[0,1]$. Consider the operator $\boldsymbol{P}$ defined for bounded Borel-measurable functions $f$ on $(-\infty, \infty)$ by

$$
P f(x)=f(x+a) p(x)+f(x-b) q(x), \quad x \in(-\infty, \infty) .
$$

This is a Markov operator corresponding to the transition probability function $P(x, B)=P 1_{B}(x)$ for $x \in(-\infty, \infty)$ and $B$ in the class $\mathscr{B}$ of Borel subsets of $(-\infty, \infty)$. A probability measure $\mu$ on $\mathscr{B}$ is said to be invariant if $\mu(B)=\int \mu(d x) P(x, B)$ for all $B \in \mathscr{B}$. The following is an extension of a result due to Frank Norman and J. W. Pickands III [1, p. 219, Theorem 4.2].

THEOREM. If $a / b$ is irrational and $0<p<1$, then there is at most one invariant probability measure.

Proof. Assume that $a / b$ is irrational and that $0<p<1$. We shall show first that any invariant probability is Lebesgue continuous. To this end, let $\mu$ be an invariant probability on $(-\infty, \infty)$. By a harmless rescaling we arrange that $a+b=1$. Let $\left\{X_{n}: n \geqslant 1\right\}$ be the Markov process on $(-\infty, \infty)$ with initial distribution $\mu$ and transition probability $p(x, E)$. Since $\mu$ is invariant, $\left\{X_{n}: n \geqslant 1\right\}$ is stationary. Let $f(x)=\exp (2 \pi i x)$ for $x$ in $(-\infty, \infty)$. Then $f$ is a map of $(-\infty, \infty)$ onto the 1-torus $T$ which is certainly measurable relative to the Borel subsets $\Re(T)$ of $T$. Let $Z_{n}=f\left(X_{n}\right)$. Since $a+b=1,\left\{Z_{n}\right.$ : $n \geqslant 1\}$ is a deterministic Markov process with $Z_{n+1}=Z_{n} e^{2 \pi i a}$. The $\left\{Z_{n}\right\}$

Received by the editors June 12, 1978 and, in revised form, October 4, 1978.

AMS (MOS) subject classifications (1970). Primary 28A65, 47A35, 60J05, 92A25.

Key words and phrases. Invariant probability, unique ergodicity, Markov operator, random ergodic theory, learning model. 
process is stationary by virtue of the stationarity of the $\left\{X_{n}\right\}$ process so the initial distribution must be $\lambda$, the Lebesgue measure on $T$. Thus $\lambda(B)=$ $\mu\left(f^{-1}(B)\right)$ for all $B$ in $\mathscr{B}(T)$. Let $n$ be any integer and suppose $A$ is a Borel subset of $[n, n+1]$ with $\mu(A)>0$. Then $\lambda(f(A))=\mu\left(f^{-1} f(A)\right) \geqslant \mu(A)>0$. But $\lambda(f(A))=m(A)$ where $m$ denotes Lebesgue measure on $(-\infty, \infty)$. Since this holds for all $n, \mu(B)>0$ implies $m(B)>0$; that is, $\mu \ll m$ and so there is a Borel measurable $g \geqslant 0$ with $\mu(B)=\int_{b} g d m$ for all $B$ in $\mathscr{B}(-\infty, \infty)$.

Suppose $\mu_{1}$ and $\mu_{2}$ are distinct invariant probabilities. Then the signed measure $\mu_{1}-\mu_{2}$ is nonzero and invariant. The positive and negative parts of $\mu_{1}-\mu_{2}$ are each invariant and are mutually singular. So if there is more than one invariant probability we can produce a pair of invariant Lebesgue continuous probabilities which are mutually singular. Thus we can obtain two measurable support sets $A$ and $B$ both of positive (though possibly infinite) Lebesgue measure. Now the support of an invariant probability $\mu$ has the property that $\mu$-a.e. point of supp $\mu$ remains in supp $\mu$ forever. Then we can pick $A^{\prime}$ in $A$ to be of finite positive Lebesgue measure so that no point of $A^{\prime}$ ever hits $B$. Thus $A^{\prime}+r a-s b \cap B$ is empty for all positive integer pairs $(r, s)$. Let $f$ be an $L_{1}$ (Lebesgue) function strictly positive on $B$ and vanishing off of $B$. Then $1_{A^{\prime}} * f$ is continuous and not identically zero. However this function vanishes on a translate of the dense set $\left(r a-s b: r, s\right.$ in $\left.Z_{+}\right)$and the contradiction finishes the argument.

Remarks. In [1, p. 217] and with greater generality in [2] Frank Norman gives sufficient conditions for the existence of invariant probabilities in terms of expected step size.

There is a generalization of this result to several terms and several dimensions (unfortunately simultaneously). The $n$-torus with a Kronecker irrational homeomorphism plays the role of the irrational rotation of $T$. We spare the reader the details.

\section{REFERENCES}

1. M. F. Norman, Markov processes and learning models, Academic Press, New York, 1972.

2. ___ Markovian learning processes, SIAM Rev. 16 (1974), 143-162.

Department of Mathematics, State University of New York at Albany, Albany, New YORK 12203

Department of Mathematics, University of Rhode Island, Kingston, Rhode Island 02881 